\title{
MOLECULAR TARGETED THERAPIES FOR PANCREATIC CANCER
}

\author{
Daniel Borja-Cacho, M.D. ${ }^{1}$, Eric Hans Jensen, M.D. ${ }^{1}$, Ashok Kumar Saluja, Ph.D. ${ }^{1}$, Donald J \\ Buchsbaum, Ph.D. ${ }^{2}$, and Selwyn Maurice Vickers, M.D. ${ }^{*}$ \\ 1 Department of Surgery, University of Minnesota, Minneapolis, MN. \\ 2Department of Radiation Oncology, University of Alabama at Birmingham, Birmingham, AL.
}

\begin{abstract}
Background-Pancreatic cancer cells express different mutations that increase the aggressiveness and confer resistance to conventional chemo- and radiotherapy. Molecules that selectively bind and inhibit these mutations are effective in other solid tumors and are now emerging as a complementary therapy in pancreatic cancer. The objective of this review is to describe the effect of drugs that inhibit specific mutations present in pancreatic cancer with special emphasis in clinical trials.
\end{abstract}

Data sources-We reviewed the English-language literature (Medline) addressing the role of drugs that target mutations present in pancreatic cancer. Both preclinical and clinical studies were included.

Conclusions-The preclinical evidence supports the combination of conventional approved therapies plus drugs that block EGFR, VEGF or induce apoptosis. However, most of the current clinical evidence is limited to small phase I trials evaluating the toxicity and safety of these regimens. The results of additional randomized trials that are still undergoing will clarify the role of these drugs in pancreatic cancer.

Mini-abstract-The role of molecular targeting in the treatment of pancreatic cancer is expanding. In this review, we summarize the most promising therapeutic targets as well as the current status of ongoing clinical trials.

\section{Keywords}

Pancreatic cancer; Epidermal Growth Factor Receptor; Vascular Endothelial Growth Factor; Death Receptor; Molecular targeted therapy

\begin{abstract}
Advances in screening, perioperative care, chemo- and radiotherapy have decreased the mortality of most cancers during the last three decades. However, the impact of these advances in pancreatic cancer is minimal. The expected incidence of pancreatic cancer in 2007 is 37,170 cases with 33,370 deaths. ${ }^{1,2}$ Pancreatic adenocarcinoma presents as a localized, regional, or systemic disease in $10-20,40$ and $40-50 \%$ of the patients respectively. ${ }^{3-5}$ The only potential curative treatment offered to patients is a combination of complete tumor removal and adjuvant therapy but less than $15 \%$ of patients present with resectable disease. ${ }^{6}$ The median survival
\end{abstract}

*Corresponding Author: Selwyn M Vickers MD, MMC no 195, 420 Delaware Street SE, Minneapolis, MN 55455, Office Phone: +1 612-626-1999, Fax number: +1 612-626-0654, Email: vickers@umn.edu.

Publisher's Disclaimer: This is a PDF file of an unedited manuscript that has been accepted for publication. As a service to our customers we are providing this early version of the manuscript. The manuscript will undergo copyediting, typesetting, and review of the resulting proof before it is published in its final citable form. Please note that during the production process errors may be discovered which could affect the content, and all legal disclaimers that apply to the journal pertain. 
after surgery is between $15-18$ months because most patients develop recurrences $9-15$ months after surgery. ${ }^{7}$ Some centers have reported a 5 year survival rate as high as $41 \%$ when complete removal of the tumor is combined with intraoperative chemotherapy 8 , however the 5-year survival in most centers after surgery for small, localized, well-differentiated tumors without lymph node invasion or positive margins is $18-24 \%$ when the tumors arise in the head or neck of the pancreas, and less than $15 \%$ when the tumor is located in the body or tail. ${ }^{9}$,

${ }^{10}$ Even in patients surviving 5 years after surgery, the next 5 -year actuarial survival (10 years after surgery) is decreased. ${ }^{11}$.

The 5-year survival for all stages is less than 5\%. ${ }^{12}$ The poor outcome and high recurrence after surgery clearly indicate the need for additional treatments either with chemo-, radiotherapy, or both, but current adjuvant and neoadjuvant therapies are not effective in pancreatic cancer. ${ }^{13,14}$ The high intrinsic resistance to chemotherapy and radiation is multifactorial. Multiple mutations that trigger proto-oncogene expression or inactivate tumor suppressor genes as well as resistance to apoptosis are important mechanisms of treatment resistance. Some common mutations present in pancreatic cancer are listed in Table 1. In an effort to improve the response to current therapies, numerous studies are combining conventional modalities with specific molecular targets. These selected therapies should increase the effectiveness of treatments without an increase in toxicity.

The objective of this review is to examine some of the current molecular therapies against pancreatic cancer. These modalities include: inhibition of growth factor receptors; induction of apoptosis; reestablish the activity of tumor suppressor genes; suppress the expression of oncogenes; and transduction signals or cell cycle modification.

\section{Therapies directed against the Epidermal Growth Factor Receptor (EGFR)}

The EGFR family is a group of transmembrane proteins that regulate key processes in the cell, such as proliferation, division, migration, and differentiation. This family has 4 different members: the EGFR (HER-1 or ErbB1), HER-2 (ErbB2), HER-3 (ErbB3) and HER-4 (ErbB4); all of them share a similar structure. ${ }^{15}$ EGF receptors have an extracellular ligand-binding domain, a hydrophobic transmembrane region, and an intracellular tyrosine kinase domain. 16 EGFR family members are frequently overexpressed in epithelial tumors (e.g. non-small cell lung cancer, breast, colorectal, renal, gastric, ovarian, and pancreatic cancer). Each member has a different frequency of expression in pancreatic tumor samples; HER-1, HER-2 and HER-3 are overexpressed in 30-60\%, 10-82\% and 60\%, respectively. ${ }^{17,18}$ High levels of EGFR in pancreatic adenocarcinoma are associated with an early aggressive disease, radiotherapy resistance, decreased survival, and poor prognosis. ${ }^{19-21}$ High HER-2 expression is associated with drug resistance, metastasis, and decreased patient survival. ${ }^{15}$ HER-3 levels are usually present in advanced stages and correlate with a decrease in postoperative survival. 22 In contrast to the other EGFR family members, HER-4 expression is more frequent in primary, non-metastatic tumors. It has been hypothesized that HER-4 expression may protect from metastasis development. ${ }^{23}$

EGFR activation in pancreatic cancer results from different mechanisms: high receptor density, EGFR activating mutations, increased ligand production, ligand-independent receptor activation, gene amplification, or loss of normal regulatory mechanisms. ${ }^{24}$ EGFR has different possible ligands, but EGF and transforming growth factor alpha (TGF $\alpha$ ) are more active in pancreatic cancer. TGF- $\alpha$ is the main EGFR ligand in vitro for pancreatic cancer cells. Pancreatic tumors coexpress high levels of ligands that activate cells either by an autocrine or paracrine effect. ${ }^{25}$ These cancer-derived growth factors can be stored in the stroma around pancreatic cancer and become released after pancreatic cancer cells produce matrix metalloproteinases. 26 
The ligand-receptor binding induces conformational changes that allow an association between two members of the EGFR family that form a homo- or heterodimer complex, and that also activate the tyrosine kinase domain. The tyrosine kinase domain recruits and phosphorylates different downstream effectors members of the Ras/PI3K pathways that amplify the initial signal. HER-2 is the most frequent receptor known to form a dimer with EGFR. ${ }^{15}$

The Ras-pathway contains several protein kinases of the Raf-family. Raf-1, the main Ras effector, activates Mitogen-Activated Protein Kinases / Extracellullar-Signal Regulated Kinases (MAPK/ERK) by phosphorylation. Activated MAPK/ERK translocate into the nucleus and enhance the expression of factors that control cell proliferation. ${ }^{27}$ Mutations in K-Ras pathway are frequent among smokers; $90-95 \%$ of patients with pancreatic cancer have this mutation. ${ }^{28}$

The second downstream pathway depends upon Phosphatidylinositol 3 Kinase (PI3K) activation. PI3K pathway controls the progression of the cell cycle, cell growth, and metabolism. This enzyme is activated directly by the tyrosine kinase domain of the EGFR or indirectly by Ras. PI3K phosphorylates and activates Akt; Akt decreases the expression of proapoptotic genes and activates IKB kinase. ${ }^{29}$ Normally inactive NFKB interacts in the cytoplasm

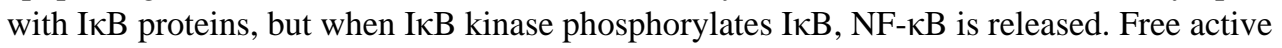
$\mathrm{NF}-\mathrm{\kappa B}$ migrates into the nucleus, inducing the transcription of different anti-apoptotic genes such as COX-2, Inhibitor of Apoptosis Proteins (IAP), X-linked Inhibitor of Apoptosis Proteins (XIAP), survivin, Bcl-xL, Bcl-2 and FLICE-like Inhibitory Protein (FLIP). ${ }^{30}$ EGFR activation also suppresses the expression of pro-apoptotic proteins (e.g. Bax levels). NF- $\kappa B$ also modifies other properties of the cell. NF- $\mathrm{KB}$ increases tumor growth, motility, invasiveness, and metastasis because NF- $\kappa B$ induces Matrix metalloproteinase-9 (MMP-9) and Urokinase Plasminogen Activator (uPA) gene transcription. 19, 31 EGF promotes the synthesis of other growth factors and cytokines (VEGF and IL- 8$)^{32}$ and allows cancer cells to resist adjuvant therapies because it increases the repair of the DNA damage induced by chemo-or radiotherapy. 21

EGFR inhibition is emerging as a promising therapy for some epithelial tumors including pancreatic cancer. Two different categories of drugs inhibit EGFR activation: the first group contains anti-EGFR antibodies that block the activation of the receptor; the second group decreases the activity of tyrosine kinase domain by preventing EGFR autophosphorylation. Figure 1 shows the EGFR activation cascade and the potential therapeutic targets that affect EGFR signaling.

\section{a) Monoclonal antibodies against EGFR}

There are several anti-EGFR monoclonal antibodies: Cetuximab (IMC-C225), Matuzumab (EMD 72000), Panitumumab (ABX-EGF), Nimotuzumab (h-R3), Zalutumumab (2F8), and ch806. Some of these compounds have been approved by the FDA for cancer treatment.

Cetuximab-The most frequent anti-EGFR antibody used against cancer is cetuximab. This chimeric, competitive, anti-EGFR antibody has a high affinity for the extracellular domain. Cetuximab limits the Ras/PI3K transduction signals. It increases the response in vitro to doxorubicin, cisplatin, 5-FU, gemcitabine, paclitaxel, irinotecan, and topotecan, among different tumors. It is effective in head and neck tumors and colorectal cancer. 33 , 34 The effectiveness of cetuximab against pancreatic cancer has been tested both in vitro and in vivo. Through EGFR binding, cetuximab induces the internalization and degradation of the receptor complex in some pancreatic cancer cell lines. It also enhances the synthesis of pro-apoptotic proteins and reduces angiogenesis by decreasing VEGF and IL- 8 synthesis. ${ }^{35}$ Cetuximab also suppresses the expression of matrix metalloproteinases (MMP). It has minimal direct cytotoxic effect in pancreatic cancer cell lines. EGFR inhibition decreases DNA repair after chemo- and 
radiotherapy, therefore cetuximab increases the effectiveness of radiation and cytotoxic drugs. Previous phase I studies in other epithelial tumors show that cetuximab can be safely combined with different chemotherapeutic agents. ${ }^{36}$ A multicenter phase II study enrolled 41 patients with locally advanced, metastatic, or recurrent pancreatic cancer. The overall survival and progression free survival rate after treatment with cetuximab and gemcitabine were higher than the historical responses with gemcitabine alone: $76 \%$ of patients experienced disease control; the median time to progression and median overall survival were 3.8 and 7.1 months respectively. Skin toxicity was the most frequent side effect, but the acne-like rash induced by cetuximab seems to correlate positively with the clinical response. EGFR levels did not predict the response. ${ }^{37}$

Combined therapy with cetuximab, gemcitabine and oxalaplatin was evaluated in a phase II study. This trial included 64 patients with metastatic pancreatic cancer, only 34 patients can be evaluated because 26 patients are still under treatment and 4 had incomplete treatment. The overall response in the evaluable patients is $38 \%$ with a median time to progression of 155 days and a preliminary 6 -month survival estimation of $54 \%$. These results encouraged a phase III study comparing cetuximab with gemcitabine. This study is still ongoing. ${ }^{38}$

Unfortunately, not all patients with pancreatic cancer respond to cetuximab therapy, and there are no useful predictors of response prior to therapy. Several mechanisms may contribute to this of response. Cetuximab is not effective against all mutant forms of EGFR. EGF receptors can form different homo- or heterodimers, but cetuximab is not active among all dimer combinations. In addition, not all pancreatic cancer cell lines internalize and degrade the receptor. ${ }^{17}$ Cetuximab has minimal effect in the transduction signals induced by HER-3. ${ }^{15}$ Mutations in the K-Ras pathway confer abnormal non-regulated activation; this effect is independent of the EGFR status. ${ }^{39,} 40$

Matuzumab-Matuzumab has similar anti-EGFR affinity, as compared to cetuximab. Preclinical evidence with this drug shows a decrease in tumor growth rate in vivo, inhibition of angiogenesis, and antibody- dependent cellular cytotoxicity in vitro. ${ }^{41}$ Most clinical studies evaluating matuzumab are phase I studies in patients with different epithelial tumors (NSCLC, colorectal, and head and neck tumors). ${ }^{42}$ The safety of matuzumab therapy in these studies encouraged phase II studies. The safety after combined therapy with matuzumab plus gemcitabine was evaluated in a phase I trial which included patients with advanced pancreatic cancer. This study showed minimal adverse effects when matuzumab and gemcitabine are used together. Although it was not the primary endpoint, according to the authors there was a partial response or stable disease present in 8 of 12 patients included in this trial. ${ }^{43}$

There are no published data evaluating anti-EGFR antibodies like ABX-EGF (Panitumumab), h-R3 (Nimotuzumab), 2F8 (Zalutumumab) and ch806 as a therapy for pancreatic cancer.

\section{b) Tyrosine Kinase Inhibitors (TKI)}

TKI were approved by FDA for treatment of patients with NSCLC, head and neck tumors, gastrointestinal stromal tumors (GIST), and more recently, pancreatic cancer. TKI are small molecules that inhibit the activity of the tyrosine kinase domain. First-line TKI are reversible, competitive inhibitors of the EGFR tyrosine kinase domain; secondline TKI are irreversible inhibitors. Second-line TKI block the EGFR tyrosine kinase domain but they also affect the tyrosine kinase domain in other receptors (e.g. HER-2, HER-4 and VEGFR). ${ }^{44}$

Most of the EGFR TKI used in clinical trials for pancreatic cancer are members of the first generation (gefitinib and erlotinib). Second generation TKI that are under evaluation are EKB-569, HKI-272, CI-1033 and ZD6474. 
Gefitinib-Gefitinib was the first EGFR TKI approved by FDA for cancer therapy because it improves local control and survival in patients with head and neck tumors. Gefitinib is also an effective therapy for NSCLC, especially among non-smoking women with welldifferentiated adenocarcinomas. ${ }^{39}$

Gefitinib decreases VEGF and factor VIII levels in pancreatic cancer in vitro. ${ }^{45}$ In contrast to anti-EGFR monoclonal antibodies, gefitinib has an inhibitory effect in cell growth in vitro and in vivo that is dose-dependent. TKI do not exhibit antibodydependent cellular toxicity, but like other EGFR inhibitors, gefitinib is a good chemoand radiosensitizer because it suppresses DNA-repair induced after these treatments. ${ }^{45}$ Clinical data evaluating gefitinib therapy in pancreatic cancer were disappointing in two phase I trials. The first study evaluated gefitinib plus capecitabine and radiotherapy; this combination was highly toxic among ten and six patients with pancreatic and rectal cancer respectively. ${ }^{46}$ The second phase I study evaluated gefitinib plus gemcitabine and radiotherapy; it was less toxic than the previous combination but its effectiveness in locally advanced pancreatic cancer was low. ${ }^{45}$

Erlotinib-Erlotinib is the only TKI inhibitor approved by FDA for patients with advanced pancreatic cancer that have not received previous chemotherapy. Erlotinib decreases ERK1/2 phosphorylation and enhances apoptosis in pancreatic cancer cells when it is combined with gemcitabine. ${ }^{47}$ Erlotinib also inhibits cell growth in pancreatic cancer cells. ${ }^{25}$ The clinical safety and effectiveness of erlotinib in cancer has been assessed in many trials. ${ }^{48}$ A phase I study evaluated the safety of erlotinib plus gemcitabine in 14 patients with unresectable pancreatic cancer; the disease control rate was $70 \%$ with acceptable toxicity.

A phase III double-blind, placebo-controlled study among 569 patients with locally advanced and metastatic pancreatic cancer showed a median survival of 6.24 months in the erlotinib/ gemcitabine group as compared with 5.91 months in the erlotinib/placebo group $(\mathrm{p}=0.038)$. The 1 -year survival rate was $23 \%$ versus $17 \%(\mathrm{p}=0.023)$. The overall disease control rate was $55.7 \%$ on erlotinib/gemcitabine versus $49.2 \%$ on placebo and gemcitabine. $(\mathrm{p}=.07)$. EGFR levels in tumor samples did not correlate with the response to erlotinib. Erlotinib induces skin toxicity, but similar to other anti-EGFR drugs, there is a positive association between the presence of rash and response. In this study, patients that received erlotinib plus gemcitabine had a higher frequency of Interstitial Lung Disease-like Syndrome as compared with both drugs administered alone. ${ }^{49}$ This study led to approval by the FDA.

\section{Mechanisms of resistance to TKI}

The clinical response to TKI in other tumors such as NSCLC is associated with specific EGFR mutations (single missense mutations or in-frame deletions). These mutations stabilize the binding of EGFR to ATP (increasing the activity of the tyrosine kinase domain) or to gefitinib (enhancing the response to the drug). ${ }^{50}$ Eighty percent of pancreatic tumor samples express EGFR, but the mutations associated with good response in other epithelial tumors are present in less than $5 \%$ of pancreatic cancer samples. ${ }^{51-53}$ The low incidence of these mutations explains the intrinsic resistance of pancreatic cancer to TKI.

Other mutations also contribute to TKI resistance. K-Ras mutations induce high levels of constitutively activated downstream signals, independently of the EGFR status. Phosphatase and Tensin Homolog (PTEN) status is also important to resistance; this phosphatase inactivates phosphorylated AKT (p-AKT). PTEN-defective cells have higher levels of active p-AKT unresponsive to EGFR inhibition. 29

Erlotinib exhibits different pharmacokinetics in smokers as compared with non-smokers. Current smokers have increased clearance of erlotinib; this effect is probably a consequence of the enzymatic induction phenomenon related to tobacco. ${ }^{54}$ 
Despite the fact that a recent study including 34 primary cell lines derived from pancreatic, colorectal, and NSCLC showed that the response to erlotinib is higher when cells coexpress EGFR and HER-3, there is no clinical or pathological useful tool to predict response in the clinical setting. 55

\section{Therapies directed against VEGF}

Angiogenesis provides the required substrates for tumor growth and dissemination. VEGF is the most important factor inducing angiogenesis, but EGF, TGF $\alpha$, FGF, and IL-8 also contribute to this process both in vivo and in vitro. ${ }^{32}$ Targeting angiogenesis is attractive because in theory it can reduce cancer progression. Different drugs inhibit angiogenesis. These drugs include MMP inhibitors, COX-2 inhibitors, thalidomide, mammalian target of rapamycin and EGFR inhibitors, but VEGF inhibitors are the most effective in pancreatic cancer. 56

The VEGF family has six different family members (VEGF, VEGF-B, VEGF-C, VEGFD, VEGF-E and placental growth factor). VEGF also known as VEGF-A is the predominant member. It has two different receptors, VEGF-R1 (Flt-1) and VEGF-R2 (Flk-1/ KDR). Both receptors have 3 different domains; the extracellular domain has seven immunoglobulin-like regions, the second and third domains constitute the transmembrane region and the intracellular tyrosine kinase domain, respectively. ${ }^{57}$ VEGF-receptor is similar to EGFR because after it binds to its ligand, the VEGF-receptor forms a dimer, the tyrosine kinase becomes autophosporylated and amplifies the initial signal by activating the intracellular pathways such as MAPK and PI3K. ${ }^{32}$ VEGF exerts most of its neoangiogenic effects through VEGFR2. ${ }^{57}$ VEGF and VEGF-R are coexpressed in $88-93 \%$ of the patients with pancreatic adenocarcinoma. 58,59 VEGF presence in tumor samples correlates with tumor size. VEGF and Microvessel Density (MVD) are also predictors of early recurrence after surgery, the development of liver metastasis, poor prognosis, and cancer-related death. ${ }^{60}$ The effects of VEGF are shown in Figure 1.

VEGF-C shares $30 \%$ homology with VEGF-A. It is specific to the lymphatic system. It induces lymphatic endothelial cell proliferation and hyperplasia of the lymphatic vasculature. VEGF$\mathrm{C}$ expression correlates with lymph node metastasis in breast, prostate, ovarian, gastric, esophageal, colorectal, gallbladder, melanoma, and pancreatic cancer. ${ }^{61}$ VEGF-D has similar structure and biological functions to VEGF-C. ${ }^{62}$ The expression of VEGF-C, VEGF-D and VEGF-C Receptor (VEGF-R3 or flt-4) is increased in pancreatic cancer. 61,62 VEGF-C and VEGF-D levels are increased in positive lymph nodes and the marginal portion of the tumor as compared with the central portion of primary pancreatic tumors. These two growth factors promote lymphatic metastasis because they induce lymphangiogenesis and facilitate the entrance of cells to the lymphatic system. High VEGF-C and VEGF-D levels correlate with worse prognosis. ${ }^{61}$ Treatment with VEGF-R Antagonists or anti-VEGF antibodies is under examination for many solid tumors. Anti-VEGF therapy does not affect cell proliferation of primary pancreatic cancer cell lines in vitro, although it does decrease endothelial cell proliferation, local infiltration, tumor growth, and metastasis in vivo.

\section{a) Anti-VEGF Antibodies}

Different antibodies with anti-VEGF activity have been evaluated for use against pancreatic cancer. Bevacizumab is a human monoclonal anti-VEGF antibody that prevents VEGF binding to both VEGF receptors. $2 \mathrm{C} 3$ is a murine antibody with antihuman VEGF activity that prevents binding of VEGF to VEGF-R2 without preventing binding to VEGF-R1. 60

Bevacizumab-Bevacizumab is the only anti-VEGF antibody that has been used in clinical trials. It increases survival, response, and progression-free survival in patients with metastatic 
colorectal cancer. ${ }^{63}$ Bevacizumab also improves the response and survival in patients with breast cancer, NSCLC, and renal cell carcinoma. ${ }^{64}$

Bevacizumab decreases lymphangiogenesis and neovascularization in vivo; it increases the delivery of chemotherapy to the tumor in pancreatic cancer. Gemcitabine plus bevacizumab were combined in a phase II study with 52 patients with stage IV pancreatic cancer. 19\% of the patients had a partial response while $48 \%$ had a stable disease. The overall median survival was 8.8 months with a median time to progression of 5.4 months and a 6 month survival of $77 \%$. The major concern with bevacizumab therapy in pancreatic cancer is bleeding and perforation, it is therefore not recommended in patients with tumors invading the duodenum or other organs. 65

Two phase III studies are currently evaluating bevacizumab therapy in pancreatic cancer. BO17706 is a phase III European study comparing gemcitabine plus erlotinib versus gemcitabine and erlotinib plus bevacizumab. The Cancer and Leukemia Group B is enrolling patients for a phase III trial comparing bevacizumab and gemcitabine versus gemcitabine and placebo in pancreatic cancer. These studies will clarify the role of bevacizumab in pancreatic cancer. ${ }^{64}$

Bevacizumab and radiation can be combined with an acceptable toxicity. Therapy with bevacizumab, capecitabine and radiotherapy was safe in a phase I study, without a significant increase in toxicity. ${ }^{66}$ The Radiation Therapy Oncology Group has an ongoing phase II trial evaluating this regimen. 56

\section{b) Other anti-VEGF Therapies}

VEGF can be inhibited with VEGF antisense oligonucleotides, VEGF-directed ribozymes, VEGF fused to diphtheria toxin, oncolytic adenoviruses, and VEGF-R TKI. ${ }^{32}$ Preclinical antiVEGF therapy in pancreatic cancer models with antisense oligonucleotide AS-3 and oncolytic adenovirus replication are promising therapies as they inhibit VEGF synthesis and angiogenesis in xenograft models, but they have not yet been tried in humans. 67,68

Valatinib-Vatalanib is an inhibitor of VEGF-R tyrosine kinase. In preclinical models valatinib plus gemcitabine show a decrease in tumor growth and metastasis in orthotopic pancreatic cancer models. This combination induces apoptosis in tumor-associated endothelial cells and decreases MVD. There are no clinical studies evaluating vatalanib and pancreatic cancer. ${ }^{69}$

Sorafenib-Sorafenib is a pan-kinase inhibitor (Raf-kinase, VEGF-R2 and PDGF Receptor). It is under evaluation as a therapy for NSCLC, liver, renal cell, colorectal, breast, melanoma, and ovarian cancer. One phase I study showed good tolerance after treatment with gemcitabine and sorafenib in pancreatic cancer. $56 \%$ of the patients had stable disease, there was no partial response seen. ${ }^{70}$

\section{Mechanisms of resistance to anti-VEGF therapy}

VEGF is the most important angiogenic factor in pancreatic cancer but anti-VEGF therapy has a minimal direct cytotoxic effect in pancreatic cancer cells; the main effect is in endothelial cells. In vivo models show that bevacizumab and other anti-VEGF therapies decrease MVD and tumor growth, mainly affecting endothelial stromal cells in the tumor. ${ }^{32}$ However, other growth hormones able to induce angiogenesis are overexpressed in pancreatic cancer. These factors include EGF, Insulin-like Growth Factor-I (IGF-I), Hepatocyte Growth Factor (HGF), Fibroblast Growth Factor (FGF), Platelet Derived Growth Factor (PDGF) and TGF- $\alpha^{71}$ High levels of these ligands correlate with increased new vessel formation and poor prognosis. 
Because the current therapy only blocks VEGF, other growth factors can still induce angiogenesis in pancreatic cancer.

Mutated or overexpressed components of the intracellular pathways also contribute to resistance. As previously mentioned, NF- $\mathrm{kB}$ and components of the Ras pathway known to mediate angiogenesis are constitutively activated in pancreatic cancer.

\section{Future directions}

\section{a) Strategies against resistance}

Even though there is an initial response after anti-EGF and anti-VEGF therapies, some epithelial tumors including pancreatic cancer have intrinsic mutations that make them primarily resistant or eventually resistant during the course of the treatment. The mutations give the tumor the ability to bypass the blockage of one pathway and, since most pathways are redundant, tumors are still able to grow. Some growth factors like EGF increase the synthesis of other growth factors like VEGF. Different receptors share certain steps in downstream pathways so that despite the inhibition of one receptor, the downstream signaling will still be activated by other non-blocked growth factors. 57

This resistance is evident in NSCLC, pancreatic, colorectal, gastric, and renal cell cancer. In an effort to decrease the resistance to these targeted therapies, combinations using both antiEGFR and anti-VEGF therapy have been assayed in vitro with a better response compared to therapy with either drug alone. Clinical trials which try to support the benefits of combined therapy are under evaluation - for example bevacizumab and erlotinib for the treatment of metastatic renal cell carcinoma, NSCLC, breast, and pancreatic cancer. ${ }^{64}$

Therapy with drugs that inhibit both pathways is another possibility to diminish resistance. Vandetanib blocks the tyrosine kinase of VEGF receptor 1, 2, 3 and EGFR. Vandetanib (also known as ZD6474) inhibits growth of pancreatic cancer cell lines and increases the response in vitro to gemcitabine and radiation. 24

Most anti-EGF therapies do not target all the members of the EGFR family. Epidermal Growth Factor Receptor-Related protein (ERRP) is a peptide found to be present in normal pancreatic duct epithelium and islet cells but the expression of ERRP is decreased in pancreatic cancer. 31 Treatment with this peptide inhibits the growth of multiple pancreatic cancer cell lines, it is also effective in xenograft models. ERRP blocks the activity of TGF- $\alpha$ and EGF because it is a pan-ErbB inhibitor. ${ }^{15}$ Future studies with this pan-ErbB Inhibitor are needed, but it may be an option to suppress the activity of all receptors.

Therapy with anti-EGF, anti-VEGF and conventional chemotherapy increases some molecules such as NF- $\mathrm{KB}$. These molecules protect the cell against normal apoptosis: but as previously mentioned they also induce proliferation. ${ }^{30}$ Compounds like curcumin and genistein, inhibit this compensatory response. ${ }^{72,73}$ When these drugs are administered in combination with chemotherapy or antibodies against EGFR or VEGF they increase the response. ${ }^{30}$ Pancreatic cancer cells have also increased production of Heat Shock Proteins (HSP) that protects cancer cells from apoptosis. Inhibition of HSP70 with compounds like quercetin, triptolide or siRNA increase apoptosis. ${ }^{74}$ These therapies are all in preclinical stages.

\section{b) Therapies inducing apoptosis through Death-Receptors}

Apoptosis or programmed cell death is triggered by numerous stimuli. These stimuli induce cell death via intrinsic and extrinsic pathways which interact at different levels. The intrinsic pathway or mitochondrial pathway is mediated through the release of cytochrome $\mathrm{C}$ and the Apoptosis Inducing Factor (AIF), both molecules form the apoptosome complex which activate 
procaspase 9. The extrinsic pathway induces cell death through activation of procaspase 8 . Both pathways converge in caspase-3 activation. Caspase-3 amplifies the original apoptotic signal through cleavage of different cytoplasmic and nuclear substrates that induce the characteristic morphological features of apoptosis. ${ }^{75}, 76$ Figure 2 illustrates the extrinsic apoptotic pathway.

Tumor cells are classified according to the response triggered during the extrinsic pathway. If cell death occurs after the stimulus elicits caspase- 8 activation and is independent of the mitochondrial pathway, cells are classified as type I. If the mitochondrial response is needed for cell death, cells are classified as type II. ${ }^{77}$

\section{Anti-Death receptor antibodies}

The extrinsic pathway requires the binding of a member of the TNF receptor family to its ligand. The most important receptors in this family are Tumor Necrosis Factor Receptor 1 (TNF-R1), Fas (CD95/Apo1) and Tumor Necrosis Factor-Related Apoptosis Inducing Ligand Receptors (also known as TRAIL or Apo-2 receptors). The ligands for these receptors are also members of the TNF family; they exist either as soluble or membrane bound proteins. The first identified ligand known to induce cell death in tumors was TNF- $\alpha$. TNF- $\alpha$ has a major in vitro activity against cancer cells but systemic administration to rodents and primates leads to adverse effects such as hypotension, leukopenia, chills, thrombus formation, ischemic and hemorrhagic lesions in tissues with distributive shock and hepatic failure. ${ }^{78}$

CD95 ligand (Apo1/FAS ligand) was the second member of this family discovered. CD95 ligand binds to CD95 receptor (Apo1/FAS receptor). CD95 Receptor-CD95 binding induces apoptosis by sequential procaspase activation. CD95 ligand is a potent inductor of apoptosis in tumor cells in vitro, but it also enhances a systemic response similar to TNF- $\alpha$ The toxic response seen during systemic therapy with these factors is due to the presence of TNF- $\alpha$ and CD95 receptors in most cells including endothelial cells and macrophages. Therefore, they do not have a specific activity against tumors. ${ }^{79}$

In 1995 TRAIL, another TNF family member was discovered. ${ }^{80}$ This soluble or membrane bound protein has five different receptors. TRAIL-R1 (Death Receptor 4 or DR4) and TRAILR2 (Death Receptor 5 or DR5) trigger apoptosis because they have a cytoplasmic domain that initiates the death signal. TRAIL also binds to receptors that lack the functional cytoplasmic domain known as antagonistic or decoy receptors. Three different decoy receptors are known: osteoprotegerin, decoy receptor 1 (DcR1/TRID/TRAIL-Re), and decoy receptor 2 (DcR2/ TRAIL-R4). Osteoprotegerin exists as a soluble receptor while DcR1 and DcR2 are present in cell membranes. ${ }^{81}$ TRAIL receptors are increased in the membrane of cancer cells; normal cells do not express these receptors, and this is important compared to other TNF family members. More than 50\% of cancer cell lines tested in vitro are TRAIL- susceptible. Brain, lung, pancreatic, liver, ovary, rectum, cervix, testes, thyroid, stomach, and laryngopharynx cancers frequently overexpress DR4 and DR5. ${ }^{82}$ The high expression of death receptors in cancer cells makes TRAIL a potential selective therapeutic candidate with minimal effect on normal cells. Figure 2 shows the apoptotic pathway induced with TRAIL.

TRAIL therapy against pancreatic cancer cells lines has been extensively evaluated. ${ }^{83,84}$ The response measured by cell viability and apoptosis is cell-line-dependent, but in general all pancreatic cancer cells have a higher resistance to TRAIL as compared with other primary tumors. There is no correlation between the levels of death receptors (including decoy receptors) and TRAIL resistance. Pancreatic cancer's resistance to TRAIL is result of the aberrant activation of anti-apoptotic proteins (such as XIAP and other IAPs) that prevent caspase activation. ${ }^{83} \mathrm{NF}-\mathrm{kB}$ is overexpressed in $70 \%$ of human pancreatic cancer cell lines; NF-B inhibits apoptosis because it up-regulates the expression of anti-apoptotic proteins. 
TRAIL sensitization can be induced with proteosome inhibitors that inhibit NF-kB and with knockdown of XIAP and BCL-xL using siRNA. ${ }^{84}$

The initial enthusiasm surrounding TRAIL therapy diminished after some studies showed liver toxicity. TRAIL induces apoptosis in normal hepatocytes in vitro and diffuse liver injury in vivo. This toxicity is due to aberrant biochemical and structural properties of some tagged forms of TRAIL. When TRAIL is FLAG/Histidine tagged or leucine zipperfused, TRAIL is cytotoxic, although native TRAIL with an internal zinc atom is unlikely to be cytotoxic. 85

The concern of liver toxicity induced by TRAIL led to the development of different antibodies targeting DR4 and DR5 were developed. These agonistic anti-Death Receptor Abs induce cell death in different tumors. Although it has been proven that the expression of TRAIL decoy receptors do not correlate with TRAIL resistance, antibodies specific for DR4 and DR5 avoid decoy receptors. ${ }^{81}$ Currently one anti-DR4 monoclonal antibody and two agonistic anti-DR5 antibodies are under evaluation. The effects of these antibodies is shown if Figure 2.

Mapatumumab (EGS-ETR1) or anti-TRAIL-R1 antibody is a monoclonal antibody developed to induce apoptosis by binding to DR4. It has been used for Non-Hodgkin lymphoma therapy. 86 A recent phase I study using this antibody as a therapy for advanced solid tumors showed no significant toxicity. ${ }^{87}$ HGS-ETR2 and TRA-8 are two different monoclonal agonistic antibodies with specific anti-DR5 activity. TRA-8 increases the effectiveness of gemcitabine both in vitro and in vivo when both drugs are used to treat pancreatic cancer cell lines. ${ }^{88}$ There are no clinical studies using this antibody. There are no studies evaluating HGS-ETR2 in pancreatic cancer.

\section{CONCLUSIONS}

Pancreatic cancer cells are resistant to conventional therapies because they overexpress different mutations that prevent cell-death or the normal response to drugs and radiotherapy. The development of new molecules that block these mutations is being more attractive because blocking them will theoretically increase the response to chemoor radiotherapy. Because the resistance emerges from multiple mutations that bypass one pathway through another pathway, the best response to these drugs will be present when the new drugs block or induce the activation of different pathways through common downstream effectors. The preclinical evidence with these emerging treatments is promising because the response with combined therapy is usually more effective than single therapy. Since the current evidence is limited to small non-randomized trials or phase I studies that evaluate the safety of the compounds, the clinical utility remains to be established. Additional studies trying to identify predictors of response and that compare these new therapies with the current standard of care are needed.

\section{REFERENCES}

1. Jemal A, Siegel R, Ward E, et al. Cancer statistics 2007. CA Cancer J Clin 2007;57:43-66. [PubMed: 17237035]

2. Messick C, Hardacre JM, McGee MF, et al. Early experience with intraoperative radiotherapy in patients with resected pancreatic adenocarcinoma. Am J Surg 2008;195:308-311. [PubMed: 18207129]discussion 312

3. Krempien R, Muenter MW, Huber PE, et al. Randomized phase II--study evaluating EGFR targeting therapy with cetuximab in combination with radiotherapy and chemotherapy for patients with locally advanced pancreatic cancer--PARC: study protocol [ISRCTN56652283]. BMC Cancer 2005;5:131. [PubMed: 16219105]

4. Baxter N, Whitson BA, Tuttle TM. Trends in the Treatment and Outcome of Pancreatic Cancer in the United States. Ann Surg Oncol 2007;14:1320-1326. [PubMed: 17225980]

5. Cowgill SM, Muscarella P. The genetics of pancreatic cancer. Am J Surg 2003;186:279-286. [PubMed: 12946833] 
6. Muller MW, Friess H, Köninger J, et al. Factors influencing survival after bypass procedures in patients with advanced pancreatic adenocarcinomas. Am J Surg 2008;195:221-228. [PubMed: 18154768]

7. Han SS, Jang JY, Kim SW, et al. Analysis of long-term survivors after surgical resection for pancreatic cancer. Pancreas 2006;32:271-275. [PubMed: 16628082]

8. Ishikawa $\mathrm{O}$, Ohigashi $\mathrm{H}$, Imaoka $\mathrm{S}$, et al. Regional chemotherapy to prevent hepatic metastasis after resection of pancreatic cancer. Hepatogastroenterology 1997;44:1541-1546. [PubMed: 9427019]

9. Riall TS, Nealon WH, Goodwin JS, et al. Pancreatic cancer in the general population: Improvements in survival over the last decade. J Gastrointest Surg 2006;10:1212-1223. [PubMed: 17114008] discussion 1223-1224

10. Eloubeidi MA, Desmond RA, Wilcox CM, et al. Prognostic factors for survival in pancreatic cancer: a population-based study. Am J Surg 2006;192:322-329. [PubMed: 16920426]

11. Riall TS, Cameron JL, Killemoe KD, et al. Resected periampullary adenocarcinoma: 5-year survivors and their 6- to 10-year follow-up. Surgery 2006;140:764-772. [PubMed: 17084719]

12. Shaib YH, Davila JA, El-Serag HB. The epidemiology of pancreatic cancer in the United States: changes below the surface. Aliment Pharmacol Ther 2006;24:87-94. [PubMed: 16803606]

13. Postier RG. The challenge of pancreatic cancer. Am J Surg 2003;186:579-582. [PubMed: 14672761]

14. Bilimoria KY, Bentrem DJ, Tomlinson JS, et al. Quality of pancreatic cancer care at Veterans Administration compared with non-Veterans Administration hospitals. Am J Surg 2007;194:588593. [PubMed: 17936418]

15. Zhang Y, Banerjee S, Wang Z, et al. Antitumor activity of epidermal growth factor receptor-related protein is mediated by inactivation of ErbB receptors and nuclear factor-kappaB in pancreatic cancer. Cancer Res 2006;66:1025-1032. [PubMed: 16424038]

16. Talar-Wojnarowska R, Malecka-Panas E. Molecular pathogenesis of pancreatic adenocarcinoma: potential clinical implications. Med Sci Monit 2006;12:186-193.

17. Arnoletti JP, Buchsbaum DJ, Huang ZQ, et al. Mechanisms of resistance to Erbitux (anti-epidermal growth factor receptor) combination therapy in pancreatic adenocarcinoma cells. J Gastrointest Surg 2004;8:960-969. [PubMed: 15585383]discussion 969-970

18. Kimura K, Sawada T, Komatsu M, et al. Antitumor effect of trastuzumab for pancreatic cancer with high HER-2 expression and enhancement of effect by combined therapy with gemcitabine. Clin Cancer Res 2006;12:4925-4932. [PubMed: 16914581]

19. Zhang H, Sawada T, Komatsu M, et al. Epidermal growth factor promotes invasiveness of pancreatic cancer cells through NF-kappaB-mediated proteinase productions. Pancreas 2006;32:101-109. [PubMed: 16340751]

20. Pino MS, Shrader M, Baker CH, et al. Transforming growth factor alpha expression drives constitutive epidermal growth factor receptor pathway activation and sensitivity to gefitinib (Iressa) in human pancreatic cancer cell lines. Cancer Res 2006;66:3802-3812. [PubMed: 16585207]

21. Bowers G, Reardon D, Hewitt T, et al. The relative role of ErbB1-4 receptor tyrosine kinases in radiation signal transduction responses of human carcinoma cells. Oncogene 2001;20:1388-1397. [PubMed: 11313882]

22. Friess H, Yamanaka Y, Kobrin MS, et al. Enhanced erbB-3 expression in human pancreatic cancer correlates with tumor progression. Clin Cancer Res 1995;1:1413-1420. [PubMed: 9815939]

23. Thybusch-Bernhardt A, Beckmann S, Juhl H. Comparative analysis of the EGF-receptor family in pancreatic cancer: expression of HER-4 correlates with a favourable tumor stage. Int J Surg Investig 2001;2:393-400.

24. Bianco C, Giovannetti E, Ciardiello F, et al. Synergistic antitumor activity of ZD6474, an inhibitor of vascular endothelial growth factor receptor and epidermal growth factor receptor signaling, with gemcitabine and ionizing radiation against pancreatic cancer. Clin Cancer Res 2006;12:7099-7107. [PubMed: 17145834]

25. Durkin AJ, Bloomston PM, Rosemury AS, et al. Defining the role of the epidermal growth factor receptor in pancreatic cancer grown in vitro. Am J Surg 2003;186:431-436. [PubMed: 14599602]

26. Korc M. Pancreatic cancer-associated stroma production. Am J Surg 2007;194:S84-S86. [PubMed: 17903452]

27. Scaltriti M, Baselga J. The epidermal growth factor receptor pathway: a model for targeted therapy. Clin Cancer Res 2006;12:5268-5272. [PubMed: 17000658] 
28. MacKenzie MJ. Molecular therapy in pancreatic adenocarcinoma. Lancet Oncol 2004;5:541-549. [PubMed: 15337483]

29. Shaw RJ, Cantley LC. Ras, PI(3)K and mTOR signalling controls tumour cell growth. Nature 2006;441:424-430. [PubMed: 16724053]

30. El-Rayes BF, Ali S, Ali IF, et al. Potentiation of the effect of erlotinib by genistein in pancreatic cancer: the role of Akt and nuclear factor-kappaB. Cancer Res 2006;66:10553-10559. [PubMed: 17079479]

31. Wang Z, Sengupta R, Banerjee S, et al. Epidermal growth factor receptor-related protein inhibits cell growth and invasion in pancreatic cancer. Cancer Res 2006;66:7653-7660. [PubMed: 16885366]

32. Xie K, Wei D, Huag S. Transcriptional anti-angiogenesis therapy of human pancreatic cancer. Cytokine Growth Factor Rev 2006;17:147-156. [PubMed: 16516532]

33. Denis F, Garaud P, Bardet E, et al. Final results of the 94-01 French Head and Neck Oncology and Radiotherapy Group randomized trial comparing radiotherapy alone with concomitant radiochemotherapy in advanced-stage oropharynx carcinoma. J Clin Oncol 2004;22:69-76. [PubMed: 14657228]

34. Cunningham D, Humblet Y, Siena S, et al. Cetuximab monotherapy and cetuximab plus irinotecan in irinotecan-refractory metastatic colorectal cancer. N Engl J Med 2004;351:337-345. [PubMed: 15269313]

35. Overholser JP, Prewett MC, Hooper AT, et al. Epidermal growth factor receptor blockade by antibody IMC-C225 inhibits growth of a human pancreatic carcinoma xenograft in nude mice. Cancer 2000;89:74-82. [PubMed: 10897003]

36. Baumann M, Krause M. Targeting the epidermal growth factor receptor in radiotherapy: radiobiological mechanisms, preclinical and clinical results. Radiother Oncol 2004;72:257-266. [PubMed: 15450723]

37. Xiong HQ, Rosenberg A, LoBuglio A, et al. Cetuximab, a monoclonal antibody targeting the epidermal growth factor receptor, in combination with gemcitabine for advanced pancreatic cancer: a multicenter phase II Trial. J Clin Oncol 2004;22:2610-2616. [PubMed: 15226328]

38. Cohenuram M, Saif MW. Epidermal growth factor receptor inhibition strategies in pancreatic cancer: past, present and the future. JOP 2007;8:4-15. [PubMed: 17228128]

39. Ono M, Kuwano M. Molecular mechanisms of epidermal growth factor receptor (EGFR) activation and response to gefitinib and other EGFR-targeting drugs. Clin Cancer Res 2006;12:7242-7251. [PubMed: 17189395]

40. Ali S, El-Rayes BF, Sarkar FH, Philip PA. Simultaneous targeting of the epidermal growth factor receptor and cyclooxygenase-2 pathways for pancreatic cancer therapy. Mol Cancer Ther 2005;4:1943-1951. [PubMed: 16373709]

41. Socinski MA. Antibodies to the epidermal growth factor receptor in non small cell lung cancer: current status of matuzumab and panitumumab. Clin Cancer Res 2007;13:s4597-S4601. [PubMed: 17671148]

42. Vanhoefer U, Tewes M, Rojo F, et al. Phase I study of the humanized antiepidermal growth factor receptor monoclonal antibody EMD72000 in patients with advanced solid tumors that express the epidermal growth factor receptor. J Clin Oncol 2004;22:175-184. [PubMed: 14701780]

43. Graeven U, Kremer B, Sudhoff T, et al. Phase I study of the humanised anti-EGFR monoclonal antibody matuzumab (EMD 72000) combined with gemcitabine in advanced pancreatic cancer. $\mathrm{Br}$ J Cancer 2006;94:1293-1299. [PubMed: 16622465]

44. Sequist LV. Second-generation epidermal growth factor receptor tyrosine kinase inhibitors in nonsmall cell lung cancer. Oncologist 2007;12:325-330. [PubMed: 17405897]

45. Maurel J, Martin-Richard M, Conill C, et al. Phase I trial of gefitinib with concurrent radiotherapy and fixed 2-h gemcitabine infusion, in locally advanced pancreatic cancer. Int J Radiat Oncol Biol Phys 2006;66:1391-1398. [PubMed: 16965868]

46. Czito BG, Willett CG, Bendell JC, et al. Increased toxicity with gefitinib, capecitabine, and radiation therapy in pancreatic and rectal cancer: phase I trial results. J Clin Oncol 2006;24:656-662. [PubMed: 16446337] 
47. Ng SS, Taso MS, Nicklee T, Hedley DW. Effects of the epidermal growth factor receptor inhibitor OSI-774, Tarceva, on downstream signaling pathways and apoptosis in human pancreatic adenocarcinoma. Mol Cancer Ther 2002;1:777-783. [PubMed: 12492110]

48. Dragovich T, Huberman M, Von Hoff DD, et al. Erlotinib plus gemcitabine in patients with unresectable pancreatic cancer and other solid tumors: phase IB trial. Cancer Chemother Pharmacol 2007;60:295-303. [PubMed: 17149608]

49. Moore MJ, Goldstein D, Hamm J, et al. Erlotinib Plus Gemcitabine Compared With Gemcitabine Alone in Patients With Advanced Pancreatic Cancer: A Phase III Trial of the National Cancer Institute of Canada Clinical Trials Group. J Clin Oncol 2007;25:1960-1966. [PubMed: 17452677]

50. Lynch TJ, Bell DW, Sordella R, et al. Activating mutations in the epidermal growth factor receptor underlying responsiveness of non-small-cell lung cancer to gefitinib. N Engl J Med 2004;350:21292139. [PubMed: 15118073]

51. Kwak EL, Jankowsi J, Thayer SP, et al. Epidermal growth factor receptor kinase domain mutations in esophageal and pancreatic adenocarcinomas. Clin Cancer Res 2006;12:4283-4287. [PubMed: 16857803]

52. Rosetti, m; Tesei, A.; Ulivi, P., et al. Modulation of drug cytotoxicity by Iressa (ZD1839) in pancreatic cancer cell lines. Cancer Biol Ther 2005;4:1089-1095. [PubMed: 16082196]

53. Tzeng CW, Frolov A, Frolova N. Epidermal growth factor receptor (EGFR) is highly conserved in pancreatic cancer. Surgery 2007;141:464-469. [PubMed: 17383523]

54. Hamilton M, Wolf JL, Rusk J, et al. Effects of smoking on the pharmacokinetics of erlotinib. Clin Cancer Res 2006;12:2166-2171. [PubMed: 16609030]

55. Buck E, Eyzaguirre A, Haley JD, et al. Inactivation of Akt by the epidermal growth factor receptor inhibitor erlotinib is mediated by HER-3 in pancreatic and colorectal tumor cell lines and contributes to erlotinib sensitivity. Mol Cancer Ther 2006;5:2051-2059. [PubMed: 16928826]

56. Saif MW. Anti-angiogenesis therapy in pancreatic carcinoma. JOP 2006;7:163-173. [PubMed: 16525200]

57. Tabernero J. The role of VEGF and EGFR inhibition: implications for combining anti-VEGF and anti-EGFR agents. Mol Cancer Res 2007;5:203-220. [PubMed: 17374728]

58. Seo Y, Baba H, Fukuda T, et al. High expression of vascular endothelial growth factor is associated with liver metastasis and a poor prognosis for patients with ductal pancreatic adenocarcinoma. Cancer 2000;88:2239-2245. [PubMed: 10820344]

59. Niedergethmann M, Hildenbrand R, Wostbrock B, et al. High expression of vascular endothelial growth factor predicts early recurrence and poor prognosis after curative resection for ductal adenocarcinoma of the pancreas. Pancreas 2002;25:122-129. [PubMed: 12142733]

60. Holloway SE, Beck AW, Shivakumar L, et al. Selective blockade of vascular endothelial growth factor receptor 2 with an antibody against tumor-derived vascular endothelial growth factor controls the growth of human pancreatic adenocarcinoma xenografts. Ann Surg Oncol 2006;13:1145-1155. [PubMed: 16791450]

61. Tang RF, Wang SX, Peng L, et al. Expression of vascular endothelial growth factors A and C in human pancreatic cancer. World J Gastroenterol 2006;12:280-286. [PubMed: 16482630]

62. Kurahara H, Takao S, Maemura K, et al. Impact of vascular endothelial growth factor-C and -D expression in human pancreatic cancer: its relationship to lymph node metastasis. Clin Cancer Res 2004;10:8413-8420. [PubMed: 15623620]

63. Cohen MH, Gootenberg J, Keegan P, Pazdur R. FDA drug approval summary: bevacizumab plus FOLFOX4 as second-line treatment of colorectal cancer. Oncologist 2007;12:356-361. [PubMed: 17405901]

64. De Gramont A, Van Cutsem E. Investigating the potential of bevacizumab in other indications: metastatic renal cell, non-small cell lung, pancreatic and breast cancer. Oncology 2005;69:46-56. [PubMed: 16301835]

65. Kindler HL, Friberg G, Singh DA, et al. Phase II trial of bevacizumab plus gemcitabine in patients with advanced pancreatic cancer. J Clin Oncol 2005;23:8033-8040. [PubMed: 16258101]

66. Crane CH, Ellis LM, Abbruzzese JL, et al. Phase I trial evaluating the safety of bevacizumab with concurrent radiotherapy and capecitabine in locally advanced pancreatic cancer. J Clin Oncol 2006;24:1145-1151. [PubMed: 16505434] 
67. Saito Y, Sunamura M, Motoi F, et al. Oncolytic replication-competent adenovirus suppresses tumor angiogenesis through preserved E1A region. Cancer Gene Ther 2006;132:242-252. [PubMed: 16179928]

68. Hotz HG, Hines OJ, Masood R, et al. VEGF antisense therapy inhibits tumor growth and improves survival in experimental pancreatic cancer. Surgery 2005;137:192-199. [PubMed: 15674201]

69. Baker CH, Solorzano CC, Fidler IJ. Blockade of vascular endothelial growth factor receptor and epidermal growth factor receptor signaling for therapy of metastatic human pancreatic cancer. Cancer Res 2002;62:1996-2003. [PubMed: 11929816]

70. Siu LL, Awada A, Takimoto, et al. Phase I trial of sorafenib and gemcitabine in advanced solid tumors with an expanded cohort in advanced pancreatic cancer. Clin Cancer Res 2006;12:144-151. [PubMed: 16397036]

71. Jia Z, Zhang J, Wei D, et al. Molecular basis of the synergistic antiangiogenic activity of bevacizumab and mithramycin A. Cancer Res 2007;67:4878-4885. [PubMed: 17510417]

72. Li L, Aggarwal BB, Shishodia S, et al. Nuclear factor-kappaB and IkappaB kinase are constitutively active in human pancreatic cells, and their down-regulation by curcumin (diferuloylmethane) is associated with the suppression of proliferation and the induction of apoptosis. Cancer 2004;101:2351-2362. [PubMed: 15476283]

73. Kunnumakkara AB, Guha S, Krishnan S, et al. Curcumin potentiates antitumor activity of gemcitabine in an orthotopic model of pancreatic cancer through suppression of proliferation, angiogenesis, and inhibition of nuclear factor-kappaB-regulated gene products. Cancer Res 2007;67:3853-3861. [PubMed: 17440100]

74. Aghdassi A, Phillips P, Dudeja V, et al. Heat shock protein 70 increases tumorigenicity and inhibits apoptosis in pancreatic adenocarcinoma. Cancer Res 2007;67:616-625. [PubMed: 17234771]

75. Debatin KM, Krammer PH. Death receptors in chemotherapy and cancer. Oncogene 2004;23:29502966. [PubMed: 15077156]

76. Huerta S, Goulet EJ, Livingston EH. Colon cancer and apoptosis. Am J Surg 2006;191:517-526. [PubMed: 16531147]

77. Fulda S, Meyer E, Friesen C, et al. Cell type specific involvement of death receptor and mitochondrial pathways in drug-induced apoptosis. Oncogene 2001;20:1063-1075. [PubMed: 11314043]

78. Ghobrial IM, Witzig TE, Adjei AA. Targeting apoptosis pathways in cancer therapy. CA Cancer J Clin 2005;55:178-194. [PubMed: 15890640]

79. Ashkenazi A, Pai RC, Fong S, et al. Safety and antitumor activity of recombinant soluble Apo2 ligand. J Clin Invest 1999;104:155-162. [PubMed: 10411544]

80. Wiley SR, Schooley K, Smolak PJ, et al. Identification and characterization of a new member of the TNF family that induces apoptosis. Immunity 1995;3:673-682. [PubMed: 8777713]

81. Cretney E, Takeda K, Smyth MJ. Cancer: novel therapeutic strategies that exploit the TNF-related apoptosis-inducing ligand (TRAIL)/TRAIL receptor pathway. Int J Biochem Cell Biol 2007;39:280286. [PubMed: 17097329]

82. Ichikawa K, Liud W, Zhao L, et al. Tumoricidal activity of a novel anti-human DR5 monoclonal antibody without hepatocyte cytotoxicity. Nat Med 2001;7:954-960. [PubMed: 11479629]

83. Vogler M, Durr K, Jovanovic M, et al. Regulation of TRAIL-induced apoptosis by XIAP in pancreatic carcinoma cells. Oncogene 2007;26:248-257. [PubMed: 16832350]

84. Khanbolooki S, Nawrocki ST, Arumugam T, et al. Nuclear factor-kappaB maintains TRAIL resistance in human pancreatic cancer cells. Mol Cancer Ther 2006;5:2251-2260. [PubMed: 16985059]

85. Srivastava RK. TRAIL/Apo-2L: mechanisms and clinical applications in cancer. Neoplasia 2001;3:535-546. [PubMed: 11774036]

86. Fanale MA, Younes A. Monoclonal antibodies in the treatment of non-Hodgkin's lymphoma. Drugs 2007;67:333-350. [PubMed: 17335294]

87. Tolcher AW, Mita M, Meropol NJ, et al. Phase I pharmacokinetic and biologic correlative study of mapatumumab, a fully human monoclonal antibody with agonist activity to tumor necrosis factorrelated apoptosis-inducing ligand receptor-1. J Clin Oncol 2007;25:1390-1395. [PubMed: 17416859] 
88. DeRosier LC, Huang ZQ, Sellers JC, et al. Treatment with gemcitabine and TRA-8 anti-death receptor- $5 \mathrm{mAb}$ reduces pancreatic adenocarcinoma cell viability in vitro and growth in vivo. $\mathrm{J}$ Gastrointest Surg 2006;10:1291-1300. [PubMed: 17114015]discussion 1300 


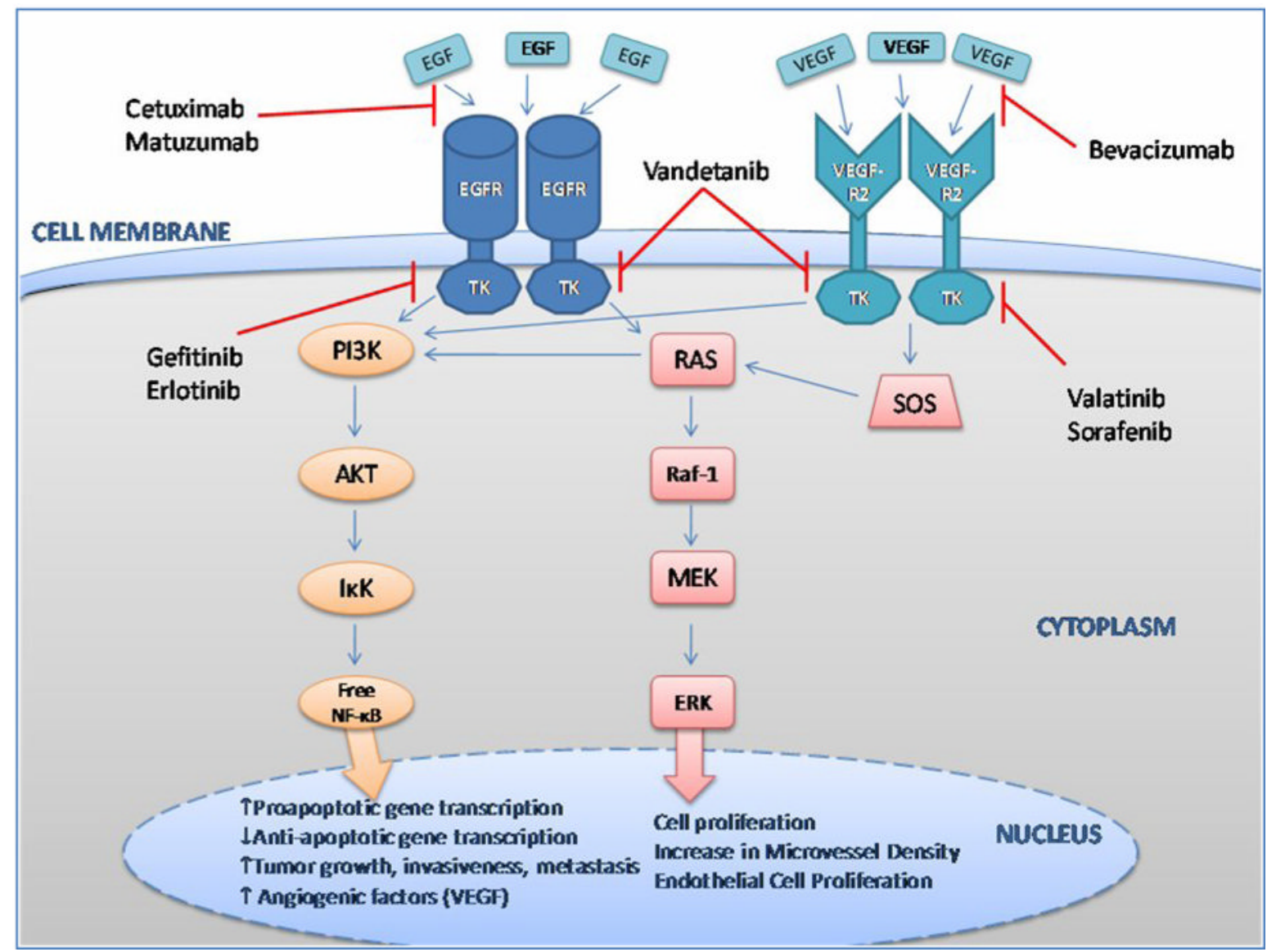

Figure 1. EGFR and VEGFR activation

After EGF binds to its receptor, conformational changes induce the activity of the Tyrosine Kinase domain (TK). This signal is amplified through the PI3K and RAS pathways leading to proliferation of cancer cells and the transcription of signals that favor the aggressiveness, survival and invasiveness. Cetuximab and Matuzumab prevent the binding of EGFR to their ligands. Gefitinib and Erlotinib inhibit the activity of the Tyrosine Kinase Domain. When VEGF binds to its receptor it also induces the formation of a dimer that triggers the activity of the Tyrosine Kinase domain. Through the RAS pathway VEGF increases the proliferation of endothelial cells. Bevacizumab prevents the binding of VEGF to its receptor. Valatinib and Sorafenib are inhibitors of the TK domain of the VEGF receptor. Vandetanib inhibits the TK domain in both VEGF and EGF receptors. 


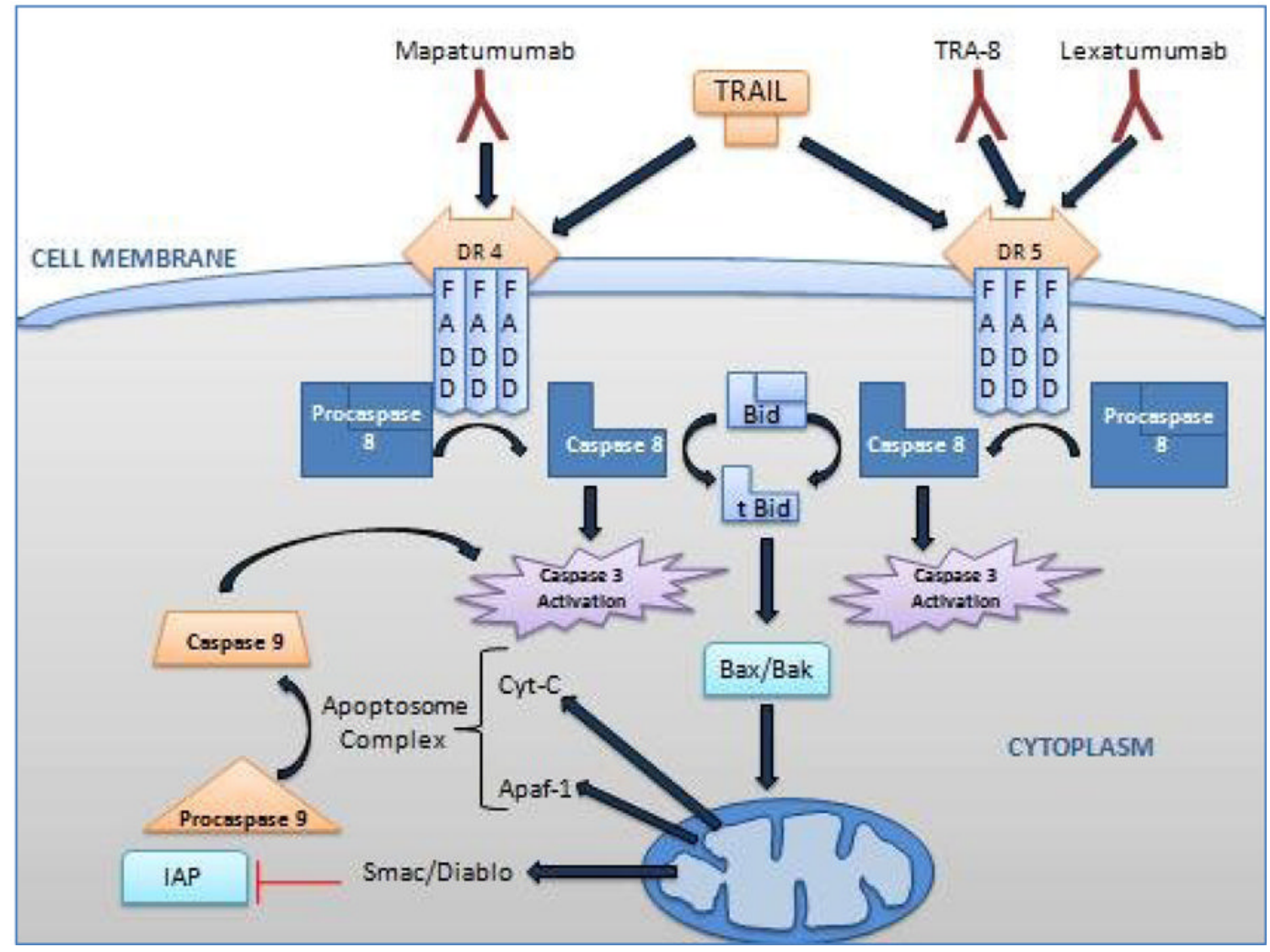

Figure 2. Apoptosis induced by TRAIL

When TRAIL binds to Death Receptor 4 (DR4) or Death Receptor 5 (DR5), the Death Inducing Signal Complex is formed. This leads to apoptosis through the extrinsic or intrinsic pathway. Mapatumumab is an agonistic antibody that triggers apoptosis through DR4. TRA-8 and Lexatumumab are two agonistic anti-DR5 antibodies that also induce apoptosis in cancer cells. 
TABLE 1

Frequency and effect of some mutations in pancreatic cance

\begin{tabular}{|c|c|c|}
\hline Mutation & Frequency & Consequence \\
\hline K-RAS mutation & $90-95 \%$ & $\begin{array}{l}\text { Resistance to apoptosis. Increase in tumor proliferation, invasiveness and } \\
\text { metastasis. }\end{array}$ \\
\hline EGFR & $30-60 \%$ & $\begin{array}{l}\text { Early aggressive disease, decreased survival and poor prognosis. Resistance to } \\
\text { chemo- and radiotherapy. }\end{array}$ \\
\hline HER-2 & $10-82 \%$ & Increase in metastasis frequency with a decrease in survival. Drug resistance. \\
\hline HER-3 & $60 \%$ & Advanced stage and decreased postoperative survival. \\
\hline VEGF & $89-93 \%$ & Early recurrence after surgery, presence of liver metastasis, poor prognosis. \\
\hline VEGF-C & $70-93 \%$ & Presence of lymph node metastasis. \\
\hline $\mathrm{NF}-\kappa \mathrm{B}$ & $70 \%$ & Upregulation of anti-apoptotic proteins. \\
\hline
\end{tabular}


Borja-Cacho et al.

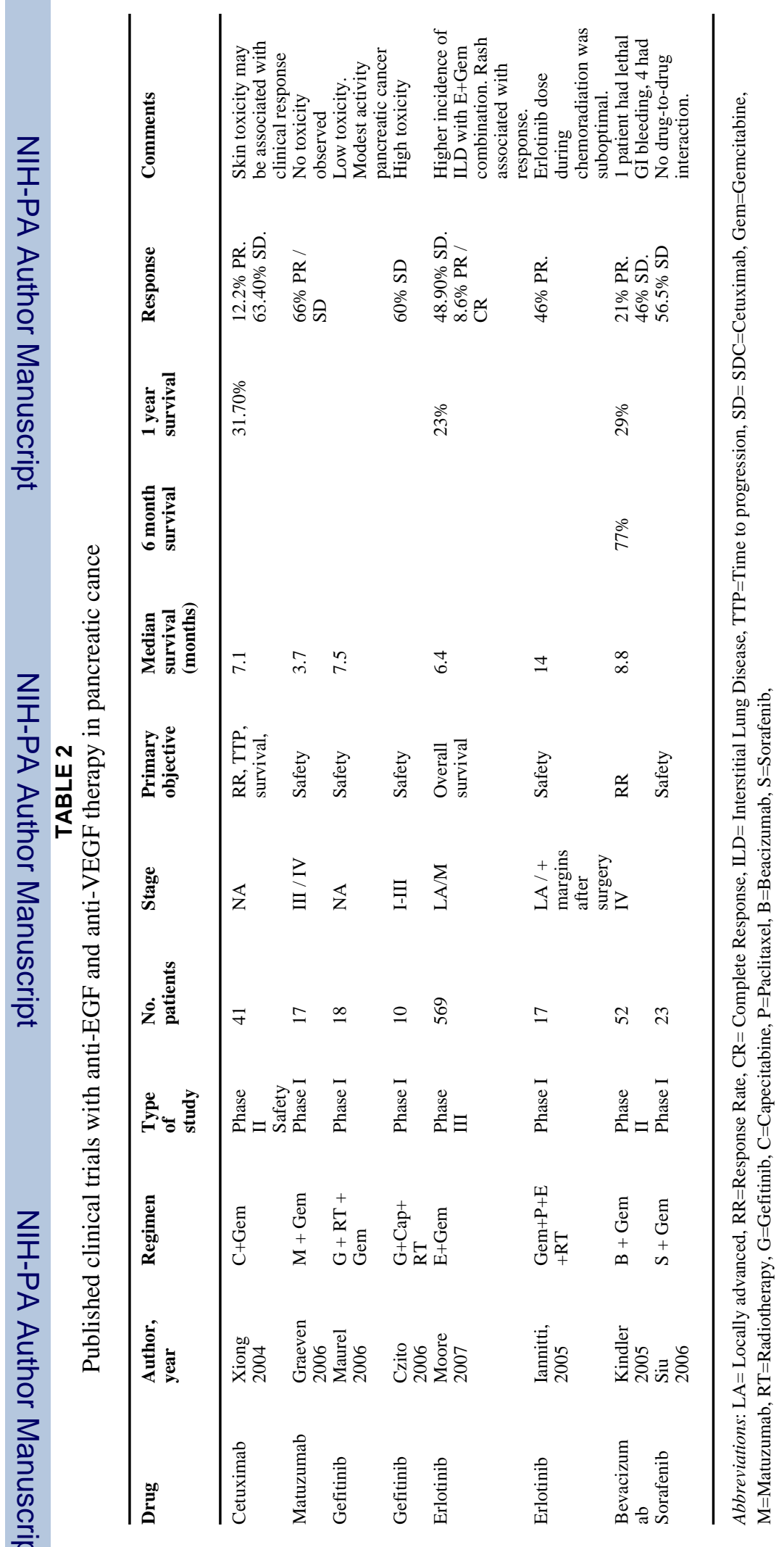

